\title{
Pattern avoidance in ascent sequences
}

\author{
Paul Duncan \\ 25 Vega Court \\ Irvine, CA 92617, USA \\ pauldncn@gmail.com
}

\author{
Einar Steingrímsson* \\ Department of Computer and Information Sciences \\ University of Strathclyde, Glasgow G1 1XH, UK \\ einar.steingrimsson@cis.strath.ac.uk
}

Submitted: Sep 16, 2011; Accepted: Nov 11, 2011; Published: Nov 21, 2011

Mathematics Subject Classification: 05A05, 05A15, 05A18, 05A19

\begin{abstract}
Ascent sequences are sequences of nonnegative integers with restrictions on the size of each letter, depending on the number of ascents preceding it in the sequence. Ascent sequences have recently been related to $(2+2)$-free posets and various other combinatorial structures. We study pattern avoidance in ascent sequences, giving several results for patterns of lengths up to 4, for Wilf equivalence and for growth rates. We establish bijective connections between pattern avoiding ascent sequences and various other combinatorial objects, in particular with set partitions. We also make a number of conjectures related to all of these aspects.
\end{abstract}

\section{Introduction and preliminaries}

An ascent sequence is a sequence $x_{1} x_{2} \ldots x_{n}$ of nonnegative integers satisfying $x_{1}=0$ and, for all $i$ with $1<i \leq n$,

$$
x_{i} \leq \operatorname{asc}\left(x_{1} x_{2} \ldots x_{i-1}\right)+1,
$$

where $\operatorname{asc}\left(x_{1} x_{2} \ldots x_{k}\right)$ is the number of ascents in the sequence $x_{1} x_{2} \ldots x_{k}$, that is, the number of places $j \geq 1$ such that $x_{j}<x_{j+1}$. An example of such a sequence is 0101312052 , whereas 0012143 is not, because the 4 is greater than $\operatorname{asc}(00121)+1=3$. Replacing $x_{j}<x_{j+1}$ in the definition of asc by $x_{j}>x_{j+1}$ gives the number of descents.

Ascent sequences became prominent after they were related to the $(2+2)$-free posets, by Bousquet-Mélou, Claesson, Dukes and Kitaev [1], who also managed to find the generating function counting these, which was quite a feat. Ascent sequences have since been studied in a series of papers by various authors, connecting them to many other combinatorial structures. These connections, and generalizations of them, have exposed what

\footnotetext{
*Steingrímsson was supported by grant no. 090038013 from the Icelandic Research Fund.
} 
seem to be deep structural correspondences in these apparently disparate combinatorial objects, including certain integer matrices, set partitions and permutations, in addition to the $(2+2)$-free posets. A good source of references and further information is [6, Section 3.2.2]; see also [3, 4, 5, 7].

In this paper we study ascent sequences avoiding certain patterns. Our patterns are analogues of permutation patterns, but they seem to provide a greater variety of counting sequences, which may perhaps be explained by the fact that ascent sequences lack the so called trivial symmetries on permutations - such as reversing a permutation to obtain another one - that imply numerical equivalence between sets of permutations avoiding different patterns.

A pattern is a word on nonnegative integers, where repetitions are allowed. An occurrence of a pattern $p$ in an ascent sequence $\mathbf{x}=x_{1} x_{2} \ldots x_{n}$ is a subsequence $x_{i_{1}} x_{i_{2}} \ldots x_{i_{k}}$ in $\mathbf{x}$, where $k$ equals the length of $p$, whose letters appear in the same relative order of size as those in $p$. For example, the ascent sequence 0123123 has three occurrences of the pattern 001, namely in the subsequences 112, 113 and 223. Note that in an occurrence of a pattern $p$, letters corresponding to two equal letters in $p$ must be equal in the occurrence, such as the 22 in 223, which correspond to the two 0's in 001. An ascent sequence $\mathbf{x}$ avoids the pattern $p$ if $\mathbf{x}$ has no occurrences of $p$. As an example, 012321 avoids 001 . If $p$ is a pattern, we let $\mathcal{S}_{p}(n)$ be the set of ascent sequences of length $n$ that avoid $p$ and $A_{p}(n)$ the number of such sequences. Also, $\mathcal{S}_{p}$ is the union of $\mathcal{S}_{p}(n)$ for all $n \geq 1$.

Since we write our patterns with nonnegative integers, whereas permutation patterns have traditionally been written with positive integers, it is important to note that the traditional permutation patterns have different names here. For example, 123 becomes 012, and 231 becomes 120. We use the latter notation, with nonnegative rather than positive integers, since ascent sequences are traditionally defined in such a way as to contain zeros.

To the best of our knowledge, pattern avoidance has not been studied for ascent sequences so far. Given the success of such studies for other combinatorial structures, such as permutations and set partitions, and the strong connections of ascent sequences to other combinatorial objects mentioned above, it is reasonable to hope for results of similar significance in the case of ascent sequences. The initial results and conjectures presented here, together with the great variety in the integer sequences counting ascent sequences avoiding various patterns, seem to indicate that this is fertile ground for interesting research.

We exhibit a connection between ascent sequences and set partitions, which does not seem to have been studied before, although such a connection, of a very different nature, is considered in [5]. A standard way to represent set partitions of the set $\{1,2, \ldots n\}$, or any other ordered set, is to write the elements of each block in increasing order, and the blocks in order of increasing minima, such as in 124-36-5, which represents the partition of $\{1,2,3,4,5,6\}$ into blocks $\{1,2,4\},\{3,6\}$ and $\{5\}$. This standard representation of a partition of $\{1,2, \ldots n\}$ can be encoded by the string $a_{1} a_{2} \ldots a_{n}$, where $a_{i}=k$ if $i$ belongs to block number $k$, counting from left to right, with the leftmost block numbered 0 . In our previous example of 124-36-5 this string would be 001021. It is easy to see that a string 
$\mathbf{x}$ of nonnegative integers encodes a set partition if and only if the first, and hence every, occurrence of each letter $k>0$ in $\mathbf{x}$ is preceded by some occurrence of $k-1$. Such a string is called a restricted growth function, or RGF. A non-crossing partition is a partition that does not have letters $a<b<c<d$ with $a, c$ in one block and $b, d$ in another.

The set of left-to-right maxima in a sequence of numbers $a_{1} a_{2} \ldots a_{n}$ is the set of $a_{i}$ such that $a_{i}>a_{j}$ for all $j<i$. The set of right-to-left minima is the set of $a_{i}$ such that $a_{i}<a_{j}$ for all $j>i$. Left-to-right minima and right-to-left maxima are defined analogously. Also, we define $\operatorname{LRmax}(\mathbf{x})$ to be the number of left-to-right maxima in a sequence $\mathbf{x}$, and LRmin, RLmax, RLmin analogously.

The Catalan numbers, which we will frequently refer to, are given by $C_{n}=\frac{1}{n+1}\left(\begin{array}{c}2 n \\ n\end{array}\right)$. The Narayana numbers are given by $N(n, k)=\frac{1}{n}\left(\begin{array}{l}n \\ k\end{array}\right)\left(\begin{array}{c}n \\ k-1\end{array}\right)$ and they refine the Catalan numbers in that $C_{n}=\sum_{k} N(n, k)$. It is well known that the Narayana numbers record the distribution of the number of ascents on permutations avoiding any given one of the patterns 132, 213, 231 and 312.

Following is an overview of our main results, which appear in Section 2. See also Table 1 at the end of the paper, where we list the counting sequences connected to our results and conjectures.

- If $p$ is any one of the patterns $10,001,010,011,012$ then $A_{p}(n)=2^{n-1}$.

- If $p$ is any one of the patterns $101,0101,021$ then $A_{p}(n)$ is the $n$-th Catalan number, and the ascent distribution on $\mathcal{S}_{p}(n)$ is given by the Narayana numbers. Moreover, the distribution of the bistatistic counting ascents and right-to-left minima is the same on $\mathcal{S}_{021}(n)$ as it is on permutations of length $n$ avoiding 132 .

- If $p$ is any one of the patterns $102,0102,0112$ then $A_{p}(n)=\left(3^{n}+1\right) / 2$.

- Ascent sequences avoiding 101 (or, equivalently, 0101) are precisely the RGFs of the non-crossing partitions.

- The set $\mathcal{S}_{p}(n)$ consists solely of RGFs if and only if $p$ is a subpattern of 01012.

In addition to the results mentioned above we conjecture, in Section 3, avoidance sequences for the patterns 210, 0123 and 0021, in terms of entries in the OnLINE ENCYClopedia of Integer Sequences [10], and also that 0021 and 1012 are Wilf equivalent, that is, have the same avoidance sequence. In particular, we conjecture that ascent sequences avoiding 210 are equinumerous with partitions avoiding 3-crossings (a 2-crossing is simply a crossing in the usual sense).

Moreover, we mention what little we know about growth rates of the counting sequences for pattern avoiding ascent sequences. In all cases we know, or conjecture, the growth is exponential, as has been proved for permutations avoiding any classical pattern [9]. Finally, we conjecture that modified ascent sequences (defined in [1]) avoiding 101 are in bijection with set partitions, and that there is a bijection taking the number of non-ascents in such a modified sequence to the number of blocks in the corresponding partition. Note that this conjecture would imply that modified ascent sequences avoiding 
101 have super-exponential growth, that is, the number of such sequences of length $n$ is not bounded by $C^{n}$ for any constant $C$.

\section{The results}

We first dispose of the easy results concerning patterns of length less than three. There is, of course, no (nonempty) ascent sequence avoiding the only pattern of length one. There are three patterns of length two, namely 00, 01 and 10. It is easy to see that the only ascent sequences avoiding 00 are the strictly increasing sequences $0123 \ldots$. one for each length $n$. There is also precisely one sequence of each length $n$ avoiding 01, namely the all zero sequence $00 \ldots 0$.

The ascent sequences avoiding 10 are precisely the weakly increasing sequences. Since, in a weakly increasing ascent sequence, each increase must be to a letter exceeding the previous maximum by one, these sequences are determined by the places of their ascents. Those places can be chosen arbitrarily among the $n-1$ slots between successive letters in a sequence of length $n$, so the number of such ascent sequences is $2^{n-1}$ and the number of

such sequences with exactly $k$ ascents is $\left(\begin{array}{c}n-1 \\ k\end{array}\right)$. The same is true of three of the sequences of length 3 as we now show.

Theorem 2.1 If $p$ is any one of the patterns 10,001,010,011, then $A_{p}(n)=2^{n-1}$. Moreover, the number of sequences in $\mathcal{S}_{p}(n)$ with $k$ ascents is $\left(\begin{array}{c}n-1 \\ k\end{array}\right)$.

Proof. We have already proved the claim for the pattern 10. A proof for each of the remaining three cases is easy to construct, given the following characterizations of sequences avoiding each one of the patterns in question:

- Ascent sequences avoiding 001 are precisely those that start with a strictly increasing sequence - necessarily $012 \ldots k$ for some $k$-followed by an arbitrary weakly decreasing sequence of letters smaller than or equal to $k$. An example is 01234444211.

- Ascent sequences avoiding 010 are precisely those that are weakly increasing and thus of the form $00 \ldots 011 \ldots 122 \ldots k k \ldots k$ for some $k$.

- Ascent sequences avoiding 011 are precisely those that consist of a strictly increasing ascent sequence arbitrarily interspersed with 0's, for example 000100230400500.

In the first two cases we use the fact that the number of weakly increasing (or weakly decreasing) sequences of nonnegative integers of length $a$, and not exceeding $b$ in value, is $\left(\begin{array}{c}a+b \\ a\end{array}\right)$.

It turns out that $A_{012}(n)$ is also $2^{n-1}$, but the ascent distribution of sequences avoiding 012 is different from the cases in Theorem 2.1.

Theorem 2.2 We have $A_{012}(n)=2^{n-1}$. The number of sequences in $\mathcal{S}_{012}(n)$ with $k$ ascents is $\left(\begin{array}{c}n \\ 2 k\end{array}\right)$. 
Proof. Ascent sequences avoiding 012 are those that have no increasing subsequence of length 3. If an ascent sequence avoids 012, then, after the initial 0 , there can be no letters other than 0 and 1, since any letter $a$ larger than 1 must be preceded by 1 , in which case we have a subsequence 01a, forming a 012. Since the initial 0 can be followed by any sequence of 0 's and 1s, the sequences avoiding 012 are precisely those that consist of an arbitrary binary string after the initial 0 , of which there are $2^{n-1}$.

To prove the result about the ascent distribution, suppose we are given a $2 k$-element subset $S=\left\{x_{1}<x_{2}<\cdots<x_{2 k}\right\}$ of the set $\{1,2, \ldots, n\}$, and we will use this to construct a binary string of length $n$, starting with 0 , with $k$ ascents. The string to be constructed will consist of 0 's in all places preceding and including place $x_{1}$, then the letters in places $x_{1}+1$ up to and including $x_{2}$ will be $1 \mathrm{~s}$. Generally, the letters in places $x_{2 i}+1$ up to and including $x_{2 i+1}$, or after place $x_{2 k}$, will be 0 's and those in places $x_{2 i+1}+1$ up to and including $x_{2 i+2}$ will be $1 \mathrm{~s}$. The sequence thus constructed will clearly have ascents precisely in the $k$ places $x_{2 i+1}$, where $i$ ranges from 0 to $k-1$. It is straightforward to construct the set $S$ of size $2 k$ from a binary string with $k$ ascents, which shows this is a bijection.

Recall that two patterns $p$ and $q$ are Wilf equivalent if $A_{p}(n)=A_{q}(n)$ for all $n$. Theorems 2.1 and 2.2 lead to the following result.

Corollary 2.3 The patterns 10,001,010,011 and 012 are Wilf equivalent.

The following lemma is interesting for its own sake, as it characterizes those patterns whose avoidance by a sequence $\mathbf{x}$ guarantees that $\mathbf{x}$ is an RGF, that is, a restricted growth function that encodes a set partition. Its second part also turns out to be useful in proving some of our other results.

Lemma 2.4 Let $p$ be a pattern. The $\mathcal{S}_{p}(n)$ consists solely of $R G F$ sequences if and only if $p$ is a subpattern of 01012 . In particular, in a sequence $\mathbf{x}$ avoiding any of these patterns, every occurrence of each letter $k \geq 1$ is preceded by some occurrence of each of the letters $0,1, \ldots, k-1$.

Proof. Clearly the sequence 01013 , not being RGF, must be excluded from any set of RGF sequences. This is guaranteed by the avoidance of $p$ only if $p$ is a pattern in 01013 , which is equivalent to being a subpattern of 01012. Thus, no other patterns than subpatterns of 01012 have avoidance sets consisting solely of RGFs. We show that the sequences avoiding any one of these patterns are RGFs, thereby establishing the claim. We prove the contrapositive, showing that if an ascent sequence is not an RGF it must contain 10102. That is sufficient, since a sequence containing 10102 of course contains all its subpatterns.

Suppose then that $\mathbf{x}$ is an ascent sequence that is not an RGF. Then there must be a leftmost letter in $\mathbf{x}$ that violates this. If that letter is $k$ then it is not preceded by $k-1$, but for some $i \geq 2$ all the letters $0,1, \ldots, k-i$ appear in $\mathbf{x}$ preceding $k$, and their first appearances are in increasing order. In order for an ascent sequence to contain $k$ but not be preceded by $k-1$, it must contain more ascents than those provided by the 
first occurrences of each of $1,2, \ldots, k-i$. Thus, one of the letters $1,2, \ldots, k-i$ appears at least twice as the rightmost letter in an ascent. Suppose this letter is $c$ and that the second one of these ascents is of the form ...ac ... Thus, the first occurrence of $c$ must precede this occurrence. But, the first occurrence of $c$ is preceded by the first occurrence of $a$. Thus, we have a subsequence $\ldots a \ldots c \ldots a c \ldots k \ldots$, and acack is an occurrence of 01012 .

The second part of the lemma follows directly from the definition of RGFs.

It turns out, as we will now show, that the ascent sequences avoiding 101 are the same as those avoiding 0101. Moreover we will show that these ascent sequences, which are RGFs according to Lemma 2.4, are precisely those that encode non-crossing partitions.

Theorem 2.5 The ascent sequences avoiding 101 are the same as those avoiding 0101, and $A_{101}(n)=A_{0101}(n)=C_{n}$, the $n$-th Catalan number. Moreover, the distribution of the number of ascents on these sequences is given by the Narayana numbers.

Proof. Clearly, an ascent sequence containing 0101 contains 101. We show that the converse is also true, thereby showing that an ascent sequence avoids 101 if and only if it avoids 0101. So, let $\ldots b \ldots a \ldots b \ldots$ be an occurrence of 101 , so $a<b$. By Lemma 2.4, since 101 is a pattern in 01012 , the first $b$ in the occurrence $\ldots b \ldots a \ldots b \ldots$ must be preceded by an occurrence of $a$. This gives the subsequence $a b a b$, which is an occurrence of 0101.

We now exhibit a bijection from 101-avoiding ascent sequences of length $n$ to 312 avoiding permutations of length $n$. Given a 101-avoiding ascent sequence $\mathbf{x}$, replace its 0 's from left to right with the numbers $k,(k-1), \ldots, 2,1$, in decreasing order, where $k$ is the number of 0 's in $\mathbf{x}$. Now repeat this for the 1 's in $\mathbf{x}$, with the numbers $(\ell+$ $k),(\ell+k-1), \ldots,(k+1)$, where $\ell$ is the number of 1 's in $\mathbf{x}$ and so on. For example, the sequence 01023200 is mapped to 45378621 . Suppose the resulting permutation $\pi$ contains an occurrence of 312 , say in a subsequence $z x y$, where $x<y<z$. Then $y$ must have come from a letter in $\mathbf{x}$ larger than the one $x$ comes from, and $z$ from a letter in $\mathbf{x}$ at least as large as the one $y$ comes from. Thus the subsequence $z x y$ in $\pi$ would correspond to a subsequence in $\mathbf{x}$ of the form $b a b$ or $c a b$, where $a<b<c$. In the first case $b a b$ is an occurrence of 101 in $\mathbf{x}$. In the second case the $c$ in $c a b$ must be preceded by $b$, by Lemma 2.4, again giving an occurrence of 101 in $\mathbf{x}$, a contradiction showing that this map produces a 312-avoiding permutation.

This map is easily seen to be invertible. Namely, given a permutation $\pi=a_{1} a_{2} \ldots a_{n}$ avoiding 312, note that if $a_{1}=k$, then the letters $k, k-1, \ldots, 1$ must appear in this decreasing order in $\pi$ for it to avoid 312. Thus, the places of these letters are filled with 0 's in the ascent sequence $\mathbf{x}$ corresponding to $\pi$. Iterating this process we next find the leftmost letter $a_{i}$ in $\pi$ that is larger than $k$ and fill the places in $\mathbf{x}$ corresponding to the places of the letters $a_{i}, a_{i}-1, \ldots, k+1$ in $\pi$ by 1 's, and so on. It is straightforward to verify that the sequence $\mathbf{x}$ thus constructed avoids 101 .

It is also easy to verify that this map preserves ascents. Thus, the 101-avoiding ascent sequences are enumerated by the Catalan numbers, and have ascent distribution given by the Narayana numbers, as is the case for 312-avoiding permutations. 
Theorem 2.6 The ascent sequences avoiding 0101 (equivalently, 101) are RGFs, and as such they encode precisely all non-crossing partitions.

Proof. Since 0101 is a pattern in 01012, the ascent sequences avoiding it are RGFs, by Lemma 2.4.

The occurrence of the pattern 0101 in an ascent sequence $\mathbf{x}$ causes a crossing, since it implies that we have letters $x y x y$, occurring in that order, in $\mathbf{x}$. Thus, if the places where these four letters occur are $a, b, c, d$, so that $a<b<c<d$, we have that $a$ and $c$ are in the same block, and $b$ and $d$ in the same block, different from the first one, which constitutes a crossing.

Conversely, suppose a set partition has a crossing consisting of letters $a<b<c<d$, with $a$ and $c$ in the same block and $b$ and $d$ together in a different block. Then either $a<b<c$ or $b<c<d$ gives an occurrence of 101, which is equivalent to having an occurrence of 0101 .

In order to give a formula for the number of ascent sequences avoiding 102 we need the following lemma.

Lemma 2.7 The number of ternary sequences of length $n$ on the letters $0,1,2$ with an even number of 2 's is $\left(3^{n}+1\right) / 2$.

Proof. It's easy to see that the number of ternary sequences of length $n$ with exactly $k$ 2 's is $\left(\begin{array}{l}n \\ k\end{array}\right) 2^{n-k}$. Thus, the difference between the number of such sequences with an even number of 2's and those with an odd number is given by

$$
(-1)^{n} \sum_{k}\left(\begin{array}{l}
n \\
k
\end{array}\right)(-2)^{n-k}=(-1)^{n}(1-2)^{n}=1 .
$$

Since the total number of ternary sequences of length $n$ is $3^{n}$, the claim follows.

Theorem 2.8 We have $A_{102}(n)=\left(3^{n-1}+1\right) / 2$.

Proof. We exhibit a bijection between sequences in $\mathcal{S}_{102}(n)$ and ternary strings of length $n-1$ with an even number of 2's. Together with Lemma 2.7 this will prove the theorem.

We will show that a 102-avoiding ascent sequence is necessarily composed of a weakly increasing ascent sequence of any length followed by a (possibly empty) sequence of what we call lifted binary strings, namely, strings composed from two integers differing in size by one. More precisely, 102-avoiding ascent sequences are those that can be written as

$$
x_{1} x_{2} \ldots x_{k} b_{1}^{1} b_{2}^{1} \ldots b_{i_{1}}^{1} b_{1}^{2} b_{2}^{2} \ldots b_{i_{2}}^{2} \ldots b_{1}^{m} b_{2}^{m} \ldots b_{i_{m}}^{m},
$$

where $x_{1} x_{2} \ldots x_{k}$ is a weakly increasing ascent sequence, the $b_{1}^{j}$ form a strictly decreasing sequence with $x_{k}>b_{1}^{1}>b_{1}^{2}>\cdots>b_{1}^{m} \geq 0$ and each $b_{p}^{q}$ equals either $b_{1}^{q}$ or $b_{1}^{q}+1$. An example of such a sequence is

$$
0012234566566564454201001 \text {, }
$$


where we have separated the respective lifted binary strings by spaces, for clarity.

It is easy to see that a sequence of this type avoids 102. We now explain why any 102-avoiding sequence has this structure.

Any ascent sequence $\mathbf{x}$ starts with a weakly increasing sequence $x_{1} x_{2} \ldots x_{k}$ of length at least 1 . In what follows, we choose $k$ such that this initial weakly increasing sequence is of maximal length. By Lemma 2.4, since 102 is a pattern in 01012, all the integers between 0 and the maximum letter in $\mathbf{x}$ must appear in $\mathbf{x}$.

If there are no letters following this initial weakly increasing subsequence of $\mathbf{x}$ then $\mathbf{x}$ clearly has the form described, with an empty sequence of lifted binary strings after the initial weakly increasing sequence. Otherwise there is a descent immediately following the initial sequence $x_{1} x_{2} \ldots x_{k}$, that is, $x_{k}$ is followed by $b_{1}^{1}$ where $x_{k}>b_{1}^{1}$. If any letter $x$ after $b_{1}^{1}$ satisfied $x>b_{1}^{1}+1$, then, for some $i$, the subsequence $x_{i}, b_{1}^{1}, x$ would form a 102 . This is because the letter $\left(b_{1}^{1}+1\right) \leq x_{k}$ must appear in the initial sequence $x_{1} x_{2} \ldots x_{k}$.

Thus, if $x_{k}$ is not the last letter of $\mathbf{x}$, then $x_{k}$ is followed by a sequence $b_{1}^{1} b_{2}^{1} \ldots b_{i_{1}}^{1}$, each of whose letters is either $b_{1}^{1}$ or $\left(b_{1}^{1}+1\right)$, and where $b_{1}^{1}<x_{k}$. An analogous argument now shows that $b_{i_{1}}^{1}$ must be followed by a sequence $b_{1}^{2} b_{2}^{2} \ldots b_{i_{2}}^{2}$ of letters $b_{1}^{2}$ and $b_{1}^{2}+1$, where $b_{1}^{2}<b_{1}^{1}$, and so on.

We now encode a 102-avoiding ascent sequence with a ternary string $\mathbf{t}=t_{2} t_{3} \ldots t_{n}$, of length $n-1$ on the symbols $0,1,2$, with an even number of 2 's. Note that the numbering of the letters in $\mathbf{t}$ starts with 2 . Recall that $k$ is the index of the last copy of the largest letter in $\mathbf{x}$, that is, the last letter of the initial weakly increasing sequence. In what follows we refer to the prefix $x_{1} x_{2} \ldots x_{k}$ of $\mathbf{x}$ as its first part, and the remainder as its second part, and likewise for the corresponding parts of $\mathbf{t}$. We let each of the letters $t_{i}$ in the first part of $\mathbf{t}$ be a 0 if $x_{i}=x_{i-1}$, and 1 otherwise, that is, if $x_{i}=x_{i-1}+1$. Some of the $1 \mathrm{~s}$ in this first part of $\mathbf{t}$ will later be changed to 2 's. We refer the reader to the example after this proof for clarification of this and the rest of the proof.

The rest of $\mathbf{t}$ is constructed from what remains of $\mathbf{x}$, that is, from the tail $x_{k+1} \ldots x_{n}$. This is the part of $\mathbf{x}$ that consists of lifted binary strings with entries that decrease in size between two consecutive such strings. Here, we let $t_{i}$ be a 2 if $x_{i}$ is the first letter $\ell$ in one of these lifted binary strings, a 0 if $x_{i}=\ell$ but is not first in the string, or a 1 if $x_{i}=\ell+1$ in the string.

We now change some of the $1 \mathrm{~s}$ in the first part of $\mathbf{t}$ to 2's as follows: Suppose the leftmost 2 in (the second part of) $\mathbf{t}$ is $t_{i}$. Then the integer $x_{i}+1$ occurs in the first part of $\mathbf{x}$, and we change $t_{j}$ from 1 to 2 , where $x_{j}$ is the leftmost occurrence of $x_{i}+1$ in $\mathbf{x}$.

The inverse of this map is described as follows, where we construct a 102-avoiding ascent sequence of length $n$ from a ternary sequence of length $n-1$, with $2 k$ copies of 2 's: Let $t_{e}$ be the $(k+1)$-st 2 in $\mathbf{t}$. Then, for $2 \leq i<e, x_{i}=x_{i-1}$ if $t_{i}=0$, and $x_{i}=x_{i-1}+1$ if $t_{i}$ is 1 or 2 . We then let $x_{e}$ be $x_{j}-1$ where $x_{j}$ is the $k$-th 2 in $\mathbf{t}$, that is, the last 2 in the first part of $\mathbf{t}$. The binary string of 0 's and 1 s strictly between $x_{e}$ and $x_{d}$, where $x_{d}$ is the next 2 in $\mathbf{t}$ after $t_{e}$, now determines the letters $x_{e+1}$ through $x_{d-1}$, where $t_{i}=0$ means $x_{i}=x_{j}-1$ and $t_{i}=1$ means $x_{i}=x_{j}$. This is now repeated for the remainder of $\mathbf{t}: x_{d}$ is set to $x_{m}-1$, where $t_{m}$ is the $k-1$-st 2 in $\mathbf{t}$, and the binary string between $t_{d}$ and the next 2 in $\mathbf{t}$ determines the corresponding letters in $\mathbf{x}$ in a way analogous to the previous 
case. This is then repeated for the rest of $\mathbf{t}$.

Here is an example of how the bijection in the proof of Theorem 2.8 works:

$$
\begin{array}{r}
\mathbf{t}=2100121022210020122 \\
\mathbf{x}=01222345567676655630
\end{array}
$$

For example, the letter below the penultimate 2 in $\mathbf{t}$ is $3=4-1$, because the second 2 in $\mathbf{t}$ is in a place where $\mathbf{x}$ has a 4 . Since the third 2 in $\mathbf{t}$ has a 6 below it in $\mathbf{x}$, the letter below the third last 2 in $\mathbf{t}$ is $6-1=5$. The letters between the 3 and the 5 in $\mathbf{x}$ mentioned here are 56 , corresponding to the 01 in the corresponding part of $\mathbf{t}$.

We next show that the patterns 102, 0102 and 0112 are Wilf equivalent.

Theorem 2.9 The patterns 102, 0102 and 0112 are Wilf equivalent. In particular, we have $\mathcal{S}_{102}(n)=\mathcal{S}_{0102}(n)$.

Proof. Of course, an occurrence of 0102 implies an occurrence of 102. It suffices to show that the converse is also true. Let bac be an occurrence of 102 in an ascent sequence $\mathbf{x}$, so $a<b<c$. Now, 102 is a subpattern of 01012, so, by Lemma 2.4, there must be an occurrence of $a$ preceding $b$, and we therefore have $a b a c$ as a subsequence in $\mathbf{x}$, constituting an occurrence of 0102 .

We next show that the number of 0112-avoiding ascent sequences of length $n$ is given by $\left(3^{n-1}+1\right) / 2$, which, together with Theorem 2.8 , completes the proof.

We claim that the ascent sequences avoiding 0112 are precisely those that consist of a strictly increasing sequence $012 \ldots k$ followed by a weakly decreasing sequence, the entire sequence arbitrarily interspersed with 0's. It is clear that a strictly increasing sequence followed by a weakly decreasing sequence cannot contain a 0112, and also that interspersing such a sequence with 0's cannot create a 0112. We thus only need to show that an occurrence of 0112 in an ascent sequence prevents it from having this prescribed form. That is straightforward, since the letter corresponding to the second 1 in such an occurrence could only belong to the weakly decreasing sequence, but that prevents it from being followed by a larger letter constituting the 2 .

To count the sequences described in the previous paragraph, observe that such a sequence is either the all zero sequence or else it consists, apart from the interspersed zeros, of a strictly increasing sequence $1,2, \ldots i$ for some $i \geq 1$, followed by a weakly decreasing sequence of letters $a_{j}$ such that $1 \leq a_{j} \leq i$. If there are $n-1-k$ zeros after the initial 0 in such a sequence $\mathbf{x}$, then we can pick their positions in $\left(\begin{array}{c}n-1 \\ n-1-k\end{array}\right)=\left(\begin{array}{c}n-1 \\ k\end{array}\right)$ ways and there are $k$ places left for the increasing and weakly decreasing sequence. Thus, the length of the increasing sequence can range from 1 to $k$. If the length of the increasing sequence is $i$ then the weakly decreasing sequence has length $k-i$ and its letters are positive and not exceeding $i$. As pointed out in the proof of Theorem 2.1, the number of weakly decreasing sequences with such parameters is $\left(\begin{array}{c}(k-i)+(i-1) \\ k-i\end{array}\right)=\left(\begin{array}{c}k-1 \\ k-i\end{array}\right)$. Thus, the total number of ascent sequences avoiding 0112 is

$$
1+\sum_{k=1}^{n-1}\left(\begin{array}{c}
n-1 \\
k
\end{array}\right) \sum_{i=1}^{k}\left(\begin{array}{l}
k-1 \\
k-i
\end{array}\right)
$$


which easily simplifies to $\left(3^{n-1}+1\right) / 2$.

We now show that ascent sequences avoiding 021 are counted by the Catalan numbers, but first a definition of a set of sequences that turn out to be equinumerous, for each $n$, with $\mathcal{S}_{021}(n)$.

Definition 2.10 $A$ restricted ascent sequence is an ascent sequence $x_{1} x_{2} \ldots x_{n}$ with $x_{i} \geq m_{i}-1$ for all $i>0$, where $m_{i}$ is the maximum value among $x_{1}, x_{2}, \ldots, x_{i-1}$. We denote the set of such sequences of length $n$ by $\mathcal{R}_{n}$.

Theorem 2.11 There is a bijection preserving ascents between $\mathcal{R}_{n}$ and $\mathcal{S}_{021}(n)$, for all $n$. Consequently, $A_{021}(n)=C_{n}$, the $n$-th Catalan number.

Proof. In a restricted ascent sequence, the only letters that can appear between two successive LR-maxima $x_{i}$ and $x_{k}$ or after its rightmost LR-maximum $x_{i}$ are $x_{i}-1$ and $x_{i}$. Moreover, any ascent sequence satisfying this condition is a restricted ascent sequence.

On the other hand, an ascent sequence $\mathbf{x}$ is easily seen to avoid 021 if and only its nonzero entries are weakly increasing. Thus, in particular, $\mathbf{x}$ is such a sequence if and only if it has just two kinds of letters, namely $x_{i}$ and 0 , between successive LR-maxima $x_{i}$ and $x_{k}$, and after its rightmost LR-maximum $x_{i}$.

Interchanging $x_{i}-1$ and 0 between successive LR-maxima in the sequences described in each of the previous two paragraphs clearly defines a bijection preserving ascents between the two sets of sequences. It is shown in Theorem 9 (due to Hilmar Gudmundsson) in [7] that $\left|\mathcal{R}_{n}\right|=C_{n}$, which completes the proof.

Theorems 2.5 and 2.11 now imply the following.

Corollary 2.12 The patterns 101, 0101 and 021 are Wilf equivalent.

An example of the bijection mentioned in the last paragraph of the proof of Theorem 2.11 is given by the following two sequences, where the top one is a restricted ascent sequence, and the bottom one a sequence avoiding 021 , and where the only differences between the two sequences are in the places of the zeros in the bottom sequence (apart from the initial zero in each):

$$
012323443465
$$

$$
012303440460
$$

Our goal is to give a bijection from the set of 021-avoiding ascent sequences to 132-avoiding permutations, and it should be noted that 021 and 132 are equivalent as patterns. Since there are very transparent bijections between restricted and 021-avoiding ascent sequences, as shown above, and between 132-avoiding and 231-avoiding permutations, by reversing each permutation, we choose to exhibit a bijection between restricted ascent sequences and 231-avoiding permutations, which is more convenient. First a definition and a lemma. 
Definition 2.13 A letter $x_{i}$ in an ascent sequence $x_{1} x_{2} \ldots x_{n}$ is maximal if $x_{i}$ is as large as it can be for its place, that is, if $x_{i}=\operatorname{asc}\left(x_{1} x_{2} \ldots x_{i-i}\right)+1$. In particular, the initial zero in an ascent sequence is defined to be maximal. If a maximal letter $x_{i}$ satisfies $x_{i}=x_{i+1}=x_{i+2}=\cdots=x_{i+k} \neq x_{i+k+1}$, or if $i+k=n$, for some $k \geq 1$, then we say that $x_{i}$ is a repeated maximal letter, and that $x_{i+k}$ is its last repetition. A maximal letter that is not repeated is its own last repetition.

For example, the maximal letters in the following ascent sequence are in bold and the last repetitions of repeated maximal letters are overlined: $00 \overline{0} 101204 \overline{4} 2320641488 \overline{8} 5$.

Lemma 2.14 In a restricted ascent sequence $\mathbf{x}=x_{1} x_{2} \ldots x_{n}$, let $x_{i}$ be the last repetition of the rightmost maximal letter in $\mathbf{x}$, and let $\mathbf{x}^{\prime}=x_{i+1} x_{i+2} \ldots x_{n}$. Then, if $\mathbf{x}^{\prime}$ is nonempty, we must have $x_{i+1}=x_{i}-1$ and the sequence obtained by subtracting $x_{i+1}$ from each letter in $\mathbf{x}^{\prime}$ is a restricted ascent sequence.

Proof. Since $x_{i}$ is the last repetition of the rightmost maximal letter, $x_{i+1}$ must be smaller than $x_{i}$. Since $\mathbf{x}$ is a restricted ascent sequence, $x_{i+1}$ cannot be smaller than $x_{i}-1$.

Now, if $k>i+1$ then $x_{k}<x_{i}+1+a$, where $a$ is the number of ascents in $\mathbf{x}^{\prime}$ that strictly precede $x_{k}$, for otherwise $x_{k}$ would be a maximal letter, contrary to the assumption about $x_{i}$. Equivalently, $x_{k} \leq x_{i+1}+1+a$. Also, $x_{k} \geq x_{i}-1=x_{i+1}$, since $\mathbf{x}$ is a restricted ascent sequence. Thus, subtracting $x_{i+1}$ from each letter in $\mathbf{x}^{\prime}$ produces a sequence of nonnegative integers that satisfies the condition defining ascent sequences. The resulting sequence is a restricted ascent sequence because subtracting the same number from each letter doesn't affect the condition for restricted ascent sequences.

As an example, the last repetition of the rightmost maximal letter in the restricted ascent sequence $\mathbf{x}=00101332232434665$ is the second 3 , so $\mathbf{x}^{\prime}=2232434665$. Reducing each letter in $\mathbf{x}^{\prime}$ by 2 we obtain 0010212443 , which is a restricted ascent sequence.

We now define a bijective map $\phi: \mathcal{R}_{n} \rightarrow \mathcal{P}_{n}(231)$, where $\mathcal{P}_{n}(231)$ is the set of 231avoiding permutations of length $n$. The map $\phi$ is defined recursively. Let $\mathbf{x}=x_{1} x_{2} \ldots x_{n}$ be a restricted ascent sequence, let the rightmost maximal letter of $\mathbf{x}$ be $m$, and suppose its last repetition is in place $p$ in $\mathbf{x}$. We then write $\mathbf{x}$ as the concatenation $\mathbf{x}=\operatorname{LmR}$, where $L=x_{1} x_{2} \ldots x_{p-1}$ and $R=x_{p+1} x_{p+2} \ldots x_{n}$. Given any sequence $\mathbf{s}$ of integers let $\mathbf{s}+t$ be the sequence obtained by adding $t$ to each of its letters and, define $\mathbf{s}-t$ analogously for subtraction. Let $\bar{R}=R-x_{p+1}$, which, by Lemma 2.14, is a restricted ascent sequence.

The permutation $\pi=\phi(\mathbf{x})$ is now constructed as the concatenation $\pi=\pi_{1} \pi_{L} \pi_{R}$. Here, $\pi_{1}$ is 1 if $m$ is a repeated maximal letter, but $\pi_{1}=p$ otherwise. We then let $\pi_{L}=\phi(L)+t$, where $t=1$ if $m$ is repeated, and $t=0$ otherwise. Also, $\pi_{R}=\phi(\bar{R})+p$. Thus, $\pi_{L}$ consists of the letters $2,3, \ldots, p$ if $m$ is repeated, but $1,2, \ldots, p-1$ otherwise, and $\pi_{R}$ consists of the letters $p+1, p+2, \ldots, n$. When $\phi$ is called recursively, $p$ and $t$ are assigned the appropriate values, which depend on which segment of $\mathbf{x}$ we are applying $\phi$ to in the recursive procedure.

As an example, we demonstrate how $\phi$ acts on the restricted ascent sequence $\mathbf{x}=$ 011213232. Note that the maximal letters in $\mathbf{x}$ are the leftmost occurrences of $0,1,2$ and 3 , with the second 1 being the only repeated maximal letter. Thus, $p=6, L=01121$, and 
$R=232$, so $\bar{R}=010$. Hence, $\pi_{1}=6, \pi_{L}=\phi(01121)+0$ and $\pi_{R}=\phi(010)+6$. Applying $\phi$ recursively gives $\pi_{L}=41325$ and $\pi_{R}=213+6=879$, so $\phi(011213232)=641325879$.

Theorem 2.15 The map $\phi$ described above is a bijection from $\mathcal{R}_{n}$ to $\mathcal{P}_{n}(231)$ and $\operatorname{asc}(\mathbf{x})=\operatorname{des}(\phi(\mathbf{x}))$ for any $\mathbf{x} \in \mathcal{R}_{n}$. Consequently, the distribution of the number of ascents on $\mathcal{S}_{021}(n)$ (or, equivalently, $\mathcal{R}_{n}$ ) is given by the Narayana numbers.

Proof. We first prove the claim $\operatorname{asc}(\mathbf{x})=\operatorname{des}(\phi(\mathbf{x}))$, then show that $\phi$ produces a 231avoiding permutation, and finally show that $\phi$ is bijective. By Theorem 2.11 the claim about ascents applies equally to sequences in $\mathcal{R}_{n}$ and $\mathcal{S}_{021}(n)$.

In the case when the rightmost maximal letter $m$ of $\mathbf{x}$ is repeated, and so is not the second letter in an ascent in $\mathbf{x}$, we have that $\pi_{1}=1$ and so $\pi_{1}$ is not the first letter in a descent in $\pi$. If $m$ is not repeated, and is not the first letter in $\mathbf{x}$, then $m$ is the second letter in an ascent in $\mathbf{x}$, and $\pi_{1}=p$, which is followed by a smaller letter than $p$, namely the first letter in $\pi_{L}$, making $\pi_{1}$ the first letter in a descent in $\pi$. Observe also that no descent can occur between $\pi_{L}$ and $\pi_{R}$ since each letter in $\pi_{L}$ is smaller than all the letters in $\pi_{R}$. Applying this argument recursively shows that $\phi$ translates ascents in $\mathbf{x}$ into descents in $\pi$, and non-ascents into non-descents.

Suppose $\pi=\phi(\mathbf{x})$ has an occurrence of 231. Such an occurrence cannot involve $\pi_{1}$, since either $\pi_{1}=1$ or else $\pi_{1}$ is larger than all the letters in $\pi_{L}$ and smaller than all the letters in $\pi_{R}$. Thus, the occurrence would have to be entirely within one of $\pi_{L}$ and $\pi_{R}$, since all the letters in $\pi_{L}$ are smaller than those in $\pi_{R}$, which prevents an occurrence of 231 from straddling the two parts. By induction we may assume that this is not the case, yielding a contradiction, so $\phi(\mathbf{x})$ must avoid 231 .

We now show that $\phi$ is injective. This is trivially true for $n=1$. Suppose $\mathbf{x}=L m R$ and $\mathbf{x}^{\prime}=L^{\prime} m^{\prime} R^{\prime}$ are restricted ascent sequences of length at least 2 , where $m$ and $m^{\prime}$ are the respective last repetitions of their rightmost maximal letters. If $\pi=\phi(\mathbf{x})=\phi\left(\mathbf{x}^{\prime}\right)$ then either both $m$ and $m^{\prime}$ are repeated maximal letters or neither is. If neither is repeated then $\pi_{1}=p>1$, where $m$ and $m^{\prime}$ are in place $p$ in $\mathbf{x}$ and $\mathbf{x}^{\prime}$, respectively. Thus, we must have that $\phi(L)=\phi\left(L^{\prime}\right)$, and $\phi(R)=\phi\left(R^{\prime}\right)$. By induction, since $L, L^{\prime}, R, R^{\prime}$ are all strictly shorter than $\mathbf{x}$, we may assume that this implies that $L=L^{\prime}$ and $R=R^{\prime}$. That, in turn, implies that $\mathbf{x}=\mathbf{x}^{\prime}$.

If $m$ and $m^{\prime}$ are repetitions of the rightmost maximal letter in $\mathbf{x}$ and $\mathbf{x}^{\prime}$, respectively, then $\pi_{1}=1$. In fact, $\pi$ then starts with $12 \ldots k$ where $k$ is the number of repetitions of the rightmost maximal letters $m$ and $m^{\prime}$, which must therefore be repeated the same number of times. Once $\phi$ has been applied successively to these repetitions we are back in the case where $m$ and $m^{\prime}$ are not repeated and, by an argument identical to the one applied to that case above, we can infer that $\mathbf{x}=\mathbf{x}^{\prime}$.

Since $\phi: \mathcal{R}_{n} \rightarrow \mathcal{P}_{n}(231)$ and $\phi$ is injective, and we know that $\mathcal{R}_{n}$ and $\mathcal{P}_{n}(213)$ are equinumerous, the map $\phi$ is a bijection.

There is an easy, and well known, bijection from 231-avoiding permutations to noncrossing partitions, that is, set partitions of $\{1,2, \ldots, n\}$ that do not contain $a<b<c<d$ with $a$ and $c$ in one block and $b$ and $d$ in another. Namely, given any permutation, split 
it into blocks after each ascent. As an example, the 231-avoiding permutation 641325879 is split into the blocks 641-32-5-87-9, or 146-23-5-78-9 in our standard notation.

It is straightforward to check that if the permutation avoids 231, then the resulting partition will have its blocks in order of increasing minima. Also, a crossing in the resulting partition would imply that there were letters $a<b<c<d$ appearing in the corresponding permutation in the order $c a d b$ or $d b c a$, but then $c d b$ or $b c a$ would have formed the pattern 231 in that permutation. With this in hand it is easy to show that when restricted to 231-avoiding permutations, this map is a bijection to non-crossing partitions.

Composing this bijection with the bijection described before Theorem 2.15 gives a bijection between non-crossing partitions and ascent sequences avoiding 021 . Using the same examples as before, we see that the 021-avoiding ascent sequence $\mathbf{x}=011213232$ corresponds to the non-crossing partition 146-23-5-78-9.

\section{$3 \quad$ Further remarks, conjectures and open problems}

For permutations, it was conjectured indepently by Stanley and Wilf, and proved by Marcus and Tardos [9], that the number of permutations of length $n$ avoiding any single pattern $p$ is bounded by $C^{n}$ for some constant $C=C(p)$ depending only on $p$. Define the growth rate of a sequence $x_{0}, x_{1}, x_{2}, \ldots$ to be the limit $\lim _{n \rightarrow \infty}\left(x_{n}\right)^{1 / n}$, if it exists. It is known that this limit always exists in the case of single patterns, so if $C(p)$ above is chosen as small as possible it is the growth rate in that case. Some work has recently been done on finding the possible growth rates of permutation classes, that is, the growth rates of sets of permutations avoiding sets of patterns, yielding very interesting results on what growth rates are possible. See [8] for a general overview, and [11] for the most recent results on permutation classes.

From the results we have presented here (see Table 1), it is straightforward to show that the growth rates for 001, 102 and 101 are 2, 3 and 4, respectively. Moreover, if Conjecture 3.3 below is true, the growth rate for 210 is 9 , as can be derived from Proposition 1 in [2]. Also, should Conjecture 3.4 hold, we would have that the growth rate for 0123 was approximately 3.247, as can be computed from the linear recurrence $a(n)=5 a(n-1)-6 a(n-2)+a(n-3)$ given for sequence A080937 in OEIS [10]. Moreover, Conjecture 3.5 implies a growth rate of 5 for 0021 (and 1012), given the recursive formula for sequence A007317 in [10].

It is a tempting conjecture that the Marcus-Tardos Theorem mentioned above also holds for ascent sequences, but this requires more work to be justified.

By reversing a 231-avoiding permutation we obtain a 132-avoiding permutation, and turn each descent into an ascent, and conversely. This, together with Theorem 2.15, shows that the number of ascents has the same distribution on 021-avoiding (a.k.a. 132-avoiding) ascent sequences and 132-avoiding permutations. We conjecture that this result can be strengthened as follows.

Conjecture 3.1 The bistatistic (asc, RLmin) has the same distribution on $\mathcal{S}_{021}(n)$ and on 132-avoiding permutations of length $n$. 
After a preliminary version of this paper was posted on the internet Bruce Sagan proved Conjecture 3.1 (personal communication).

Let fwd be the length of the maximal final weakly decreasing sequence in an ascent sequence. For example, fwd $(01123035523220)=4$, since 3220 has length 4 . are the rightmost occurrences of $0,2,3$ and 5 . We then have the following conjecture, where zeros $(\mathbf{x})$ is the number of 0 's in an ascent sequence $\mathbf{x}$.

Conjecture 3.2 We have $A_{0012}(n)=C_{n}$, the $n$-th Catalan number. Moreover, the bistatistic (asc, fwd) on $\mathcal{S}_{0012}(n)$ has the same distribution as (asc, RLmax) does on permutations avoiding the pattern 132. In particular, this implies that the number of ascents has the Narayana distribution on $\mathcal{S}_{0012}(n)$. Also, the bistatistics (asc, fwd) and (asc, zeros) have the same distribution on 0012-avoiding ascent sequences.

We should point out that the bistatistics (asc, RLmin) on 132-avoiding permutations and (asc, RLmax) on 231-avoiding permutations mentioned in Conjectures 3.1 and 3.2 have the same distribution as double upsteps and the number of returns to the $x$-axis on Dyck paths. We have found more apparent equidistributions of bistatistics of a similar kind to those in Conjectures 3.1 and 3.2, and it seems certain that many more could be found easily.

Conjecture 3.3 The number $A_{210}(n)$ equals the number of non-3-crossing set partitions of $\{1,2, \ldots, n\}$. See sequence A108304 in [10], where non-3-crossing is also defined.

Conjecture 3.4 The number $A_{0123}(n)$ equals the number of Dyck paths of semilength $n$ and height at most 5. See sequence A080937 in [10].

Conjecture 3.5 The patterns 0021 and 1012 are Wilf equivalent, and $A_{0021}(n)=$ $A_{1012}(n)$ is given by the binomial transform of Catalan numbers, which is sequence A007317 in [10].

It is easy to show that the Wilf equivalences proved or conjectured here are the only ones possible for the set of all patterns of length at most four. This is because other pairs of sequences are seen, by computer testing, to diverge.

Thus, the following are all the Wilf equivalences for patterns of length at most four, where the cases of 0012 and the pair $(0021,1012)$ depend on Conjectures 3.2 and 3.5, respectively, and the other results on Corollaries 2.3 and 2.12 and Theorem 2.9, in addition to the trivial equivalence of 00 and 01 :

$$
\begin{aligned}
& 00 \sim 01 \\
& 10 \sim 001 \sim 010 \sim 011 \sim 012, \\
& 102 \sim 0102 \sim 0112, \\
& 101 \sim 021 \sim 0101 \sim 0012, \\
& 0021 \sim 1012 .
\end{aligned}
$$


When computing the numbers of ascent sequences avoiding various pairs of patterns of lengths 3 and 4 we found several sequences of numbers recognized by the OEIS [10], such as Motzkin numbers, various transformations of the Catalan and Fibonacci numbers, and sequences counting permutations avoiding pairs of patterns. We also found some apparent Wilf equivalences that might be interesting. We do not list any of these here, but we will gladly share our data with anybody who might be interested.

Finally, we consider the modified ascent sequences defined in [1, Section 4.1]. Given an ascent sequence $\mathbf{x}$, we successively create sequences $\mathbf{x}_{1}, \mathbf{x}_{2}, \ldots, \mathbf{x}_{k}$, where $k$ is the number of ascents in $\mathbf{x}$. The sequence $\mathbf{x}_{i+1}$ is constructed from $\mathbf{x}_{i}$ by increasing by 1 each letter in $\mathbf{x}_{i}$ that precedes the $i$-th ascent in $\mathbf{x}$ and is larger than or equal to the larger letter in that ascent. The sequence $\mathbf{x}_{k}$ is then defined to be the modified ascent sequence associated to $\mathbf{x}$. Observe that in constructing each $\mathbf{x}_{i}$ we preserve the ascents in $\mathbf{x}$. It is easy to see that this is an invertible process so that modified ascent sequences are in bijection with ascent sequences. As an example, the modified ascent sequence of 010221212 successively becomes 010331212 and 010441312 , with the first two ascents causing no changes.

Conjecture 3.6 On modified ascent sequences the patterns 101,0101, 1021, 1102, 1120, 1210 are all Wilf equivalent, and the number of ascent sequences avoiding any one of these patterns is the $n$-th Bell number. These sequences are thus equinumerous with partitions of an n-element set. Moreover, the distribution of the number of ascents on such sequences is the reverse of the distribution of the number of blocks on set partitions. That is, the number of modified ascent sequences of length $n$ with $k$ ascents, and avoiding any single one of these patterns, equals the number of set partitions of an n-element set with $n-k$ blocks.

Bruce Sagan (personal communication) has proved the above conjecture in the case of the pattern 101 and $k=1$.

Note that if Conjecture 3.6 is true then the number of modified ascent sequences avoiding 101 would not have exponential growth, since that is known to be false for the Bell numbers; they grow faster than $C^{n}$ for any constant $C$.

\section{Acknowledgments}

We are grateful to Anders Claesson, Mark Dukes, Sergey Kitaev and Jeff Remmel for useful discussions and suggestions. We are also deeply indebted to a referee who corrected a few errors and made many useful suggestions, which led to a substantial improvement of the presentation. In particular, that referee pointed out the bijection now used in the proof of Theorem 2.5.

\section{References}

[1] M. Bousquet-Mélou, A. Claesson, M. Dukes and S. Kitaev: (2+2)-free posets, ascent sequences and pattern avoiding permutations, J. Comb. Theory Ser. A 117(7) (2010), 884-909. 


\begin{tabular}{|c|c|c|c|c|}
\hline Pattern $p$ & Number of sequences avoiding $p$ & OEIS & Formula & Reference \\
\hline $\begin{array}{l}001 \\
010 \\
011 \\
012\end{array}$ & $1,2,4,8,16,32,64,128,256,512, \ldots$ & A000079 & $2^{n-1}$ & Thms. 2.1, 2.2 \\
\hline $\begin{array}{c}102 \\
0102 \\
0112\end{array}$ & $1,2,5,14,41,122,365,1094,3281,9842, \ldots$ & A007051 & $\left(3^{n}+1\right) / 2$ & Thm. 2.8 \\
\hline $\begin{array}{c}101 \\
021 \\
0101\end{array}$ & $1,2,5,14,42,132,429,1430,4862,16796, .$. & A000108 & $\frac{1}{n+1}\left(\begin{array}{c}2 n \\
n\end{array}\right)$ & Thms. 2.5, 2.11 \\
\hline 000 & $\begin{array}{l}1,2,4,10,27,83,277,1015,4007,17047 \\
77451,374889,1923168,10427250, \ldots\end{array}$ & & & \\
\hline 100 & $\begin{array}{l}1,2,5,14,44,153,583,2410,10721,50965 \\
257393,1374187,7722862,45520064, \ldots\end{array}$ & & & \\
\hline 110 & $\begin{array}{l}1,2,5,14,43,143,510,1936,7774,32848 \\
145398,671641,3227218,16084747, \ldots\end{array}$ & & & \\
\hline 120 & $\begin{array}{l}1,2,5,14,42,133,442,1535,5546,20754 \\
80113,317875,1292648,5374073, \ldots\end{array}$ & & & \\
\hline 201 & $\begin{array}{l}1,2,5,15,52,201,843,3764,17659,86245 \\
435492,2261769,12033165,65369590, \ldots\end{array}$ & & & \\
\hline 210 & $\begin{array}{l}1,2,5,15,52,202,859,3930,19095,97566, \\
520257,2877834,16434105,965054901, \ldots\end{array}$ & A108304 & & Conj. 3.3 \\
\hline 0123 & $\begin{array}{l}1,2,5,14,42,131,417,1341,4334,14041, \\
45542,147798,479779,1557649, \ldots\end{array}$ & A080937 & & Conj. 3.4 \\
\hline $\begin{array}{l}0021 \\
1012\end{array}$ & $\begin{array}{l}1,2,5,15,51,188,731,2950,12235,51822, \\
223191,974427,4302645,19181100 \ldots\end{array}$ & A007317 & & Conj. 3.5 \\
\hline
\end{tabular}

Table 1: Number sequences for pattern avoidance by ascent sequences. All patterns of length 3 are listed, a few of length 4 . OEIS refers to entry in [10]. 
[2] M. Bousquet-Mélou and G. Xin: On partitions avoiding 3-crossings, Sém. Lothar. Combin. 54 (2006), Article B54e, 21 pp.

[3] A. Claesson and S. Linusson: $n$ ! matchings, $n$ ! posets, Proc. Am. Math. Soc. 139 (2011), 435-449.

[4] M. Dukes, J. Remmel, S. Kitaev and E. Steingrimsson: Enumerating (2+2)-free posets by indistinguishable elements, J. Comb. 2(1) (2011), 139-163.

[5] M. Dukes and R. Parviainen: Ascent sequences and upper triangular matrices containing non-negative integers. Electronic J. Combin. 17(1) (2010), \#R53 (16pp).

[6] S. Kitaev: Patterns in permutations and words, Monographs in Theoretical Computer Science (with a foreword by Jeffrey B. Remmel), Springer-Verlag, ISBN 978-3-64217332-5, 2011.

[7] S. Kitaev and J. Remmel: Enumerating $(2+2)$-free posets by the number of minimal elements and other statistics, Discrete Applied Mathematics, to appear.

[8] M. Klazar: Some general results in combinatorial enumeration (English summary), Permutation patterns, 3-40, London Math. Soc. Lecture Note Ser. 376, Cambridge Univ. Press, Cambridge, 2010.

[9] A. Marcus and G. Tardos: Excluded permutation matrices and the Stanley-Wilf conjecture, J. Combin. Theory Ser. A 107(1) (2004), 153-160.

[10] OEIS Foundation Inc. (2011), The On-Line Encyclopedia of Integer Sequences, http://oeis.org.

[11] V. Vatter: Small permutation classes, Proc. London Math. Soc., 2011, doi: 10.1112/plms/pdr017. 